\title{
Clinical Value of Transperineal 3D Volume Ultrasound Combined with 2D High Frequency Ultrasound in Anal Fistula
}

\author{
Dan Yang ${ }^{1}$, Xiufen Yao ${ }^{2 *}$ \\ ${ }^{1}$ Department of Ultrasound, Affiliated Hospital of Tibet University for Nationalities, Xianyang, China \\ ${ }^{2}$ Department of Ultrasound, Affiliated Hospital of Traditional Chinese Medicine, Xianyang, China \\ Email: ${ }^{\star} 15353768441 @ 163 . c o m$
}

How to cite this paper: Yang, D. and Yao, X.F. (2020) Clinical Value of Transperineal 3D Volume Ultrasound Combined with 2D High Frequency Ultrasound in Anal Fistula. Journal of Biosciences and Medicines, 8 , 12-19.

https://doi.org/10.4236/jbm.2020.85002

Received: March 23, 2020

Accepted: April 27, 2020

Published: April 30, 2020

Copyright $\odot 2020$ by author(s) and Scientific Research Publishing Inc. This work is licensed under the Creative Commons Attribution International License (CC BY 4.0).

http://creativecommons.org/licenses/by/4.0/

\begin{abstract}
Objective: To explore the clinical diagnostic value of transperineal volume ultrasound combined with two-dimensional high-frequency ultrasound for anal fistula. Methods: A total of 52 patients with anal fistula admitted to the Affiliated Hospital of Shaanxi University of Traditional Chinese Medicine from December 2017 to July 2018 were selected. They were all undergoing transperineal 3D volume ultrasound combined with $2 \mathrm{D}$ high-frequency ultrasound examination, and the diagnosis results were analyzed. The results of ultrasonography and surgical pathology were compared. Results: Among 52 patients, $3 \mathrm{D}$ volume ultrasound combined with $2 \mathrm{D}$ high-frequency ultrasound were used to diagnose 32 cases of anal fistula intersphincteric type, 14 cases of transsphincter type, 5 cases of supra-sphincter type, and 1 case of extra-sphincter type. T supervisor classification accuracy rate is $90 \%$. The detection rate of branch pipes was $92 \%$, and the compliance rate of internal fistula was $95 \%$. Two-dimensional high-frequency ultrasound was used to diagnose 34 cases of anal fistula intersphincteric type, 14 cases of transsphincter type, 4 cases of supra-sphincter type, and 0 cases of extra-sphincter type. The detection rate of branch canals was $42 \%$, and the accuracy of type classification was $90 \%$. The coincidence rate was $95 \%$. There was a statistically significant difference in the detection rate of the anal fistula branch and the coincidence rate of the internal fistula between the two methods (both $\mathrm{P}<0.05$ ). In the anal fistula classification, there was no significant statistical difference between the two methods. Conclusion: 1) The overall coincidence rate of three-dimensional volumetric ultrasound combined with two-dimensional high-frequency ultrasound in the diagnosis of anal fistula is high; 2) Three-dimensional volumetric ultrasound technology has great application prospects in infants and anal fistulas.
\end{abstract}




\section{Keywords}

Two-Dimensional High-Frequency Ultrasound, Anal Fistula, Three-Dimensional Volume Ultrasound Combined with

Two-Dimensional High-Frequency Ultrasound

\section{Introduction}

Anal fistula is a good disease and common disease in clinical anorectal surgery [1]. It consists of an internal fistula, an external fistula and a fistula. The internal fistula is located around the dentate line, the external fistula is located on the skin around the anus, and the fistula runs on the anus, peripheral tissue clearance. Although these patients are not life-threatening, if they are not handled properly, they can easily recur and seriously affect the quality of life of patients. Therefore, they should be treated as soon as possible after diagnosis. Surgery is the only effective way to treat such diseases. Proper handling is the key to a successful one-time operation [2]. This study evaluated the anal fistula lesions by combining three-dimensional volume ultrasound and two-dimensional high-frequency ultrasound technology (combined group), and observed whether the combined application of the two technologies for the overall diagnostic efficacy of the disease is higher than the two-dimensional high-frequency ultrasound technology alone. Provide a more valuable basis for the treatment and evaluation of anal fistula.

\section{Information and Methods}

1) Subjects 52 patients (average age of $39.4 \pm 13.5$ years [ 4 months - 63 years]) with anal fistula collected from December 2018 to June 2019 in the Department of Anorectal Surgery, Affiliated Hospital of Shaanxi University of Traditional Chinese Medicine. Inclusion criteria: a) patients with clinical findings of anal fistula requiring ultrasound examination; b) voluntary treatment and signing of surgical consent; c) complete clinical data; Exclusion criteria: a) ultrasound examination or surgery Patients who were not performed in our hospital; b) patients with incomplete clinical data; c) patients with severe cardiopulmonary diseases, such as severe hypertension, arrhythmia, coronary heart disease, etc.; d) patients with anorectal stenosis. All the patients or their relatives had previously given signed informed consent.

2) Instruments and methods: In this study, GE Voluson E8 and Voluson E10, high-frequency probe M6S; three-dimensional volume probe RAB4-8-D and RM6C were used. The operation is completed by two senior ultrasound doctors. No special intestinal preparation is required before the examination. The patient takes a lithotomy position, fully exposes the lesion, performs routine visual inspection and digital examination, and makes a preliminary judgment of the lesion. The ultrasound scans the superficial lesions as a whole, determines the location of the lesion, the position of theinternal fistula and other information, and records the 
inspection results. Then use a three-dimensional volume probe, the instrument presets pelvic floor conditions for scanning, and performs three-dimensional volume imaging on the longitudinal section of the anal canal to display information about the lesion and anal canal. Based on Render imaging, TUI and VCI tomography are performed. Adjust the layer distance, and conduct a comprehensive evaluation of the lesion information on the $\mathrm{A}$ and $\mathrm{C}$ cut faces, and then repeat the above operation on the cross section of the anal canal. The evaluation shows the depth position of the lesion, determines the type of the lesion, the position of the internal fistula, etc., and records the inspection results.

3) Anal fistula classification: According to the Parks [3] classification criteria, anal fistulas are classified as follows: a) Intersphincteric: the fistula is shaped between the internal and external sphincter, the internal fistula is around the dentate line, and the external fistula is mostly near the edge of the anus. Low anal fistula; b) Transsphincter: the fistula penetrates the internal and external sphincter of the anal canal, the sciatic rectum space, and opens on the skin of the anal canal, which can be a low anal fistula or a high anal fistula; c) Supra sphincter: the fistula passes through the anus The intrasphincteric sphincter extends upward through the anal canal sphincter, crosses the puborectalis and the external anal sphincter, and opens down to the perianal skin through the sciatic rectal space. It is a high anal fistula; d) Extra-sphincter: the fistula is from the perineal skin, go up through the sciatic rectal space and levator ani muscle, and then pierce the pelvis or rectum. The internal and external sphincter without anal canal, the internal fistula can be near the dentate line, or the rectum, often caused by trauma, Crohn, intestinal malignant tumors and other non-caused. The position of the inner port allows the error to be within one point.

4) Statistical methods were analyzed using IBM SPSS 20.0 statistical software. Comparison of three-dimensional volume ultrasound combined with two-dimensional high-frequency ultrasound, two-dimensional high-frequency ultrasound alone, and two-dimensional high-frequency ultrasound alone was compared with the main type of anal fistula, branch detection rate, and intraoral coincidence rate using paired $\chi^{2}$ test. $\mathrm{P}<0.05$ was considered statistically significant.

\section{Results}

1) Table 1 shows the results of anal fistula typing. Among them, 52 cases of anal fistula were detected by two-dimensional high-frequency ultrasound. Two cases of transsphincteric anal fistula were misdiagnosed as intersphincteric type, 2 cases of supra-sphincteric anal fistula were transsphincter type, and 1 case of extra-sphincteric anal fistula was supra-sphincter type. 47 cases were typed, and the coincidence rate was $90 \%$ (47/52). A total of 52 cases of anal fistula were detected in the combination group, 1 case was misdiagnosed as an intersphincteric type, and 1 case was misdiagnosed as a transsphincteric type. 50 cases were accurately typed, and the classification coincidence rate was $95 \%$ (50/52). There was no significant difference in the classification of the anal fistula between the two methods $\left(\chi^{2}=1.378, \mathrm{P}=0.240\right)$. 
2) The detection rate is shown in Table 2. Anal fistulas are divided into simple and complex anal fistulas. Complex anal fistulas often have 2 or more internal ports. Among the 52 cases of anal fistula, 8 patients with complicated anal fistula have 2 internal fistula. Therefore A total of 60 internal fistula were detected during surgery, and 49 were detected by two-dimensional high-frequency ultrasound. The compliance rate was $82 \%$ (49/60). 57 were detected by the joint group, with a compliance rate of $95 \%(56 / 60)$. There was a statistically significant difference in the detection rate of the anal fistula between the two methods $\left(\left(\chi^{2}=6.175, \mathrm{P}=\right.\right.$ $0.023)$.

3) The coincidence rate of the two anal fistulas detected by the two inspection methods is shown in Table 3. Of the 52 anal fistulas, 12 patients had anal fistulas. Five cases of branch tubes were detected by two-dimensional high-frequency ultrasound with a coincidence rate of $42 \%$ (5/12), and 11 cases of branch tubes were detected by the combined group with a coincidence rate of $92 \%(11 / 12)$. The difference in the detection rate of anal fistula branches between the two methods was statistically significant $\left(\left(\chi^{2}=1.378, \mathrm{P}=0.240\right)\right.$.

4) Figure 1 shows a case of intersphincteric anal fistula, which is the most common type of anal fistula. Figure 2 shows a transsphincter anal fistula, which is the second most common type of anal fistula. Both cases are low-grade simple anal fistula.

Table 1. Comparison of different types of anal fistula diagnosis and surgical examination results $(n)$.

\begin{tabular}{|c|c|c|c|c|c|c|}
\hline Methods & Numbers & Intersphincteric & Transsphincter & $\begin{array}{c}\text { Supra } \\
\text { sphincter }\end{array}$ & $\begin{array}{c}\text { Extra } \\
\text { Sphincter } \\
\text { shape }\end{array}$ & $\begin{array}{c}\text { Coincidence } \\
\text { rate }\end{array}$ \\
\hline $\begin{array}{l}\text { 2D high-frequency } \\
\text { ultrasound group }\end{array}$ & 52 & 34 & 14 & 4 & 0 & $90 \%$ \\
\hline Joint group & 52 & 32 & 14 & 5 & 1 & $95 \%$ \\
\hline Surgical diagnosis & 52 & 32 & 14 & 5 & 1 & $100 \%$ \\
\hline
\end{tabular}

Table 2. Comparison of the results of the compliance rate of the anal fistula with the three inspection methods (n).

\begin{tabular}{cccc}
\hline Methods & Numbers & Number of checkouts & Coincidence rate \\
\hline 2D high-frequency ultrasound group & 60 & 49 & $82 \%$ \\
Joint group & 60 & 57 & $95 \%$ \\
\hline
\end{tabular}

Table 3. Comparison of detection rates of anal fistula branches by three inspection methods (n).

\begin{tabular}{cccc}
\hline Methods & Numbers & Number of checkouts & Coincidence rate \\
\hline 2D high-frequency ultrasound group & 12 & 5 & $42 \%$ \\
Joint group & 12 & 11 & $92 \%$ \\
\hline
\end{tabular}




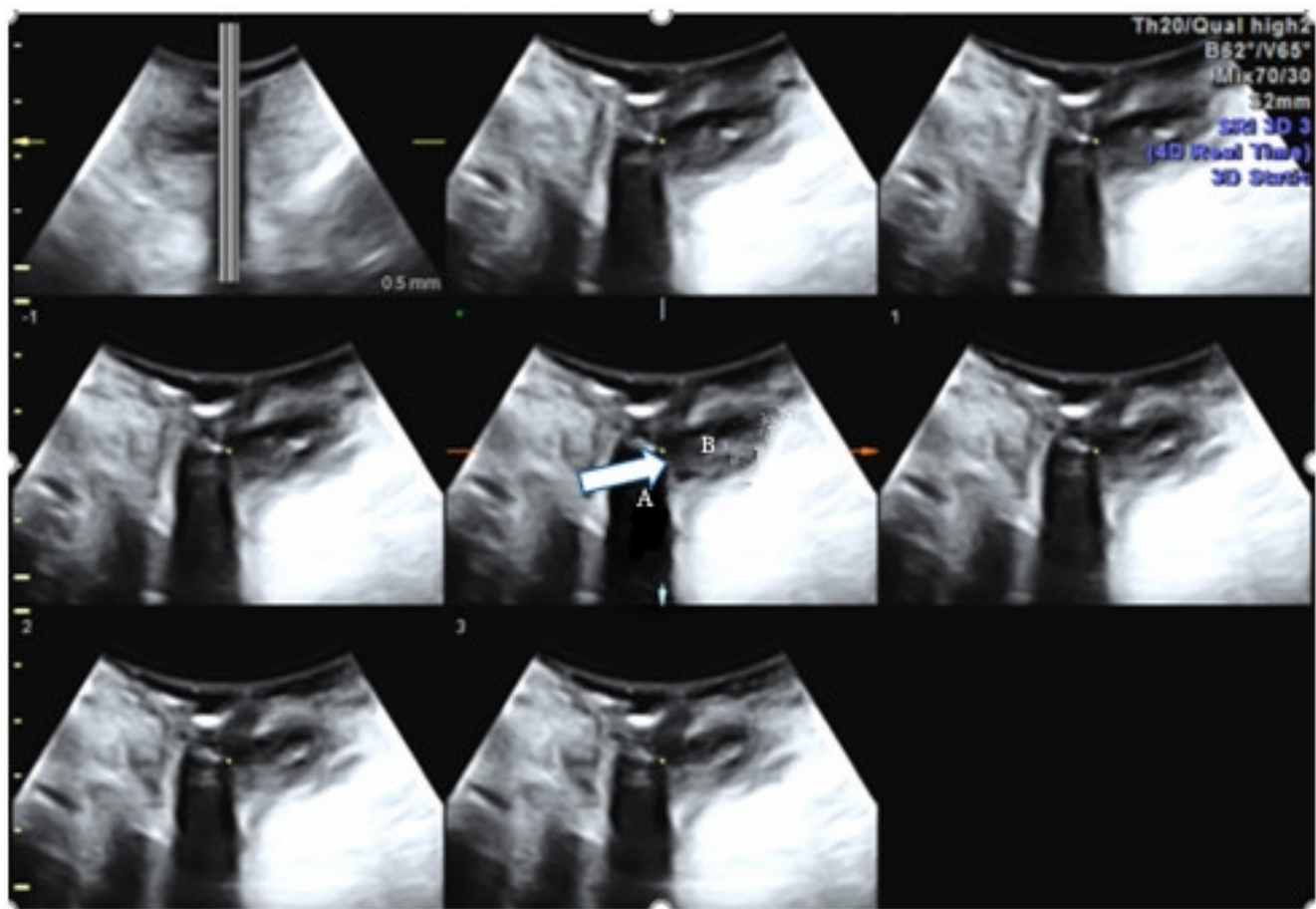

Young men, first attack. Three-dimensional volumetric ultrasound VCI technology A plane $(3 \times 3)$ of the longitudinal section of the anal canal shows the ultrasound image of the relationship between the transsphincteric fistula and the anal can$\mathrm{al}$, and a point-like strong echo can be seen at the internal fistula, and the internal fistula is located at 6 points. A is longitudinal section of anal canal; B fistula; the arrow indicates the internal fistula.

Figure 1. Intersphincteric.

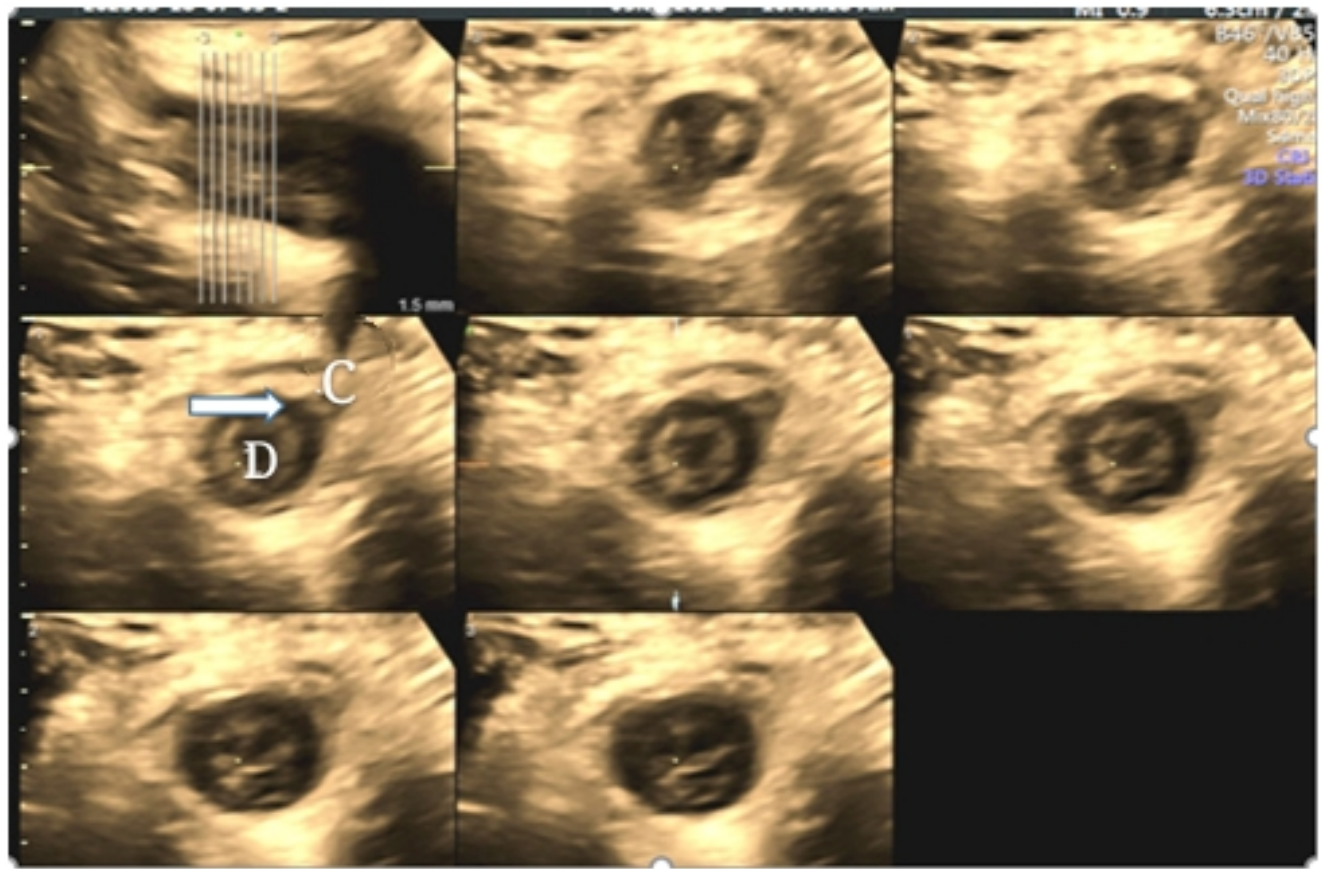

Middle-aged and elderly men with first episode. The three-dimensional volumetric ultrasound VCI technology A plane (3 $\times 3$ ) of the anal canal cross section shows the ultrasound image of the relationship between the sphincteric fistula and the anal canal, with the internal fistula at 1 point. $\mathrm{C}$ is the cross section of the anal canal; D fistula; the arrow indicates the internal fistula.

Figure 2. Transsphincter. 


\section{Discussion}

Anal fistula is a common and frequently-occurring disease in the clinic. Surgery is the only effective way to treat anal fistula. The anatomical structure of the lesion and the position of the internal fistula are the keys to the operation. Therefore, preoperative diagnosis is crucial [4]. Among the many existing examination methods, intracavitary three-dimensional ultrasound has great clinical value in the diagnosis of anal fistula [5] [6], but intracavitary three-dimensional ultrasound requires the purchase of specialized anorectal instruments and is an invasive inspection method that is not easily accepted by patients. The four-dimensional color ultrasound system currently equipped in the ultrasound room of each hospital is equipped with abdominal volume probes and superficial probes as standard. They are mostly used for prenatal diagnosis and female pelvic floor function tests [7]. This study was applied to the examination of anal fistula and gave the above results. Tomographic imaging technology TUI and volumetric imaging technology VCI under transperineal volume ultrasound can display multiple static continuous tomographic 2D images on a plane at the same time, presenting imaging images similar to CT and MRI, and VCI technology provides thick layers $(6-10 \mathrm{~mm})$ or thin layer $(1-4 \mathrm{~mm})$ imaging. The layer thickness can be adjusted to obtain accurate information about disease changes. At the same time, VCI technology has high-quality resolution and clearly displays the tissue outline. The three-dimensional display of the lesion information shows the relationship between the lesion and the anal canal, and the diagnosis result is obtained.

On the other hand, infants and young children are also prone to anal fistula due to various factors such as their anatomy, immunity, and hormone levels [8] [9]. In this group, the anus is small, shorter than adults, and the anal glands are developed. At present, there is no more suitable examination method except two-dimensional high-frequency ultrasound, but two-dimensional high-frequency ultrasound can only scan the two-dimensional information of the fistula section, and cannot evaluate the three-dimensional relationship between the fistula and the anal canal [10], three-dimensional The application of volumetric ultrasound in anal fistula can greatly make up for this deficiency, and it has become the first choice of anal fistula for infants and young children.

Foreign reports [11] [12] The accuracy of anal canal rectal cavity three-dimensional ultrasound diagnosis of anal fistula is $81.0 \%-98.5 \%$, and the accuracy of internal mouth positioning is $89.5 \%-96.4 \%$. Domestic reports [13] the correct rate of three-dimensional anal canal rectal ultrasound diagnosis of anal fistula is $96.6 \%$ - $100 \%$, the intraoral compliance rate is $91.3 \%-95 \%$, and the consistency rate of branch detection is $85 \%-100 \%$. In this study, the results of $3 \mathrm{D}$ volume ultrasound combined with $2 \mathrm{D}$ high-frequency ultrasound were consistent with reports. The detection rate of $3 \mathrm{D}$ volume ultrasound on the branch tube was slightly lower than the report, and the coincidence rate of other indicators was consistent with the report. In the classification of the anal canal, 5 cases were 
misdiagnosed by two-dimensional high-frequency ultrasound, of which 1 case was misdiagnosed as supra-sphincter type, and 2 cases were misdiagnosed as transsphincter type, both of which were tortuous and complicated due to high anal fistula. Failure to follow up continuously led to misdiagnosis. The remaining 2 cases of misdiagnosis between the lower anal fistulas were misdiagnosed as the fistula due to postoperative complications. Two cases were misdiagnosed in the combined group, and both were postoperative. The overall condition around the anal canal showed poor results, and no accurate judgment was made on the relationship between muscles. In terms of the detection rate of the internal fistula, two internal high-frequency ultrasounds failed to detect 11 internal fistula, and only one internal fistula was detected for 6 complicated anal fistulas. 6 internal fistula were missed, and 3 cases had recurrence after surgery. The scar tissue was mistakenly detected. The internal fistula was located, and three internal fistula were misdiagnosed. Two cases of anal fistula were non-acute, and the boundary between the anal fistula and surrounding tissues was unclear. The internal fistula was unclear, which caused two misdiagnoses. In the combination group, 3 were not successfully detected, 2 cases of anal fistula were non-acute, and the internal fistula was unclear, which caused misdiagnosis of 2 internal fistula, and 1 case of complex anal fistula. The patient was postoperative, and the anal canal was more severe. The overall image showed Poor, missed diagnosis of 1 internal fistula. In terms of branch tube detection rate, 7 cases of branch tubes were not successfully detected by $2 \mathrm{D}$ high-frequency ultrasound. The combined group showed better overall performance of branch tubes, and the detection rate of branch tubes was significantly better than that of $2 \mathrm{D}$ high-frequency ultrasound.

In summary, as a new ultrasound technology, transperineal volumetric ultrasound provides a non-invasive, multi-section, multi-dimensional stereoscopic display of the relationship between the lesion and the anal canal, and the specific conditions of the lesion. It is an anal fistula. The patient offers a new examination. The application of transperineal 3D volume ultrasound can greatly make up for the regret that $3 \mathrm{D}$ ultrasound in the rectum is not suitable, especially for infants and anal stenosis patients. 3D volume ultrasound has a good application in infants and anal stenosis patients with perianal disease. It has a high acceptance rate with family members, and the diagnostic coincidence rate is consistent with intracavitary ultrasound. It provides accurate information for clinicians and improves the cure rate of one-time surgery. It is worthy of widespread clinical application.

\section{Acknowledgements}

This work was supported by the Xianyang Science and Technology Plan Region (No: 2018k02-101).

\section{Conflicts of Interest}

The authors declare no conflicts of interest regarding the publication of this paper. 


\section{References}

[1] Slauf, P., Antoš, F. and Marx, J. (2014) Acute Periproctal Abscesses. Rozhledy v Chirurgii, 93, 226-231.

[2] Zhang, G.J. and Cha, J. (2016) Application Value of Transrectal Biplane Color Doppler Ultrasound in the Diagnosis of Perianal Abscess. Primary Medical Forum, 20, 1801-1802.

[3] Parks, A.G., Gordon, P.H. and Hardcastle, J.D. (1976) A Classification of Fistula-In-Ano. British Journal of Surgery, 63, 1-12. https://doi.org/10.1002/bjs.1800630102

[4] Li Y. and Li, C.F. (2017) Application Value of Transrectal Biplane Ultrasound and Transcutaneous High Frequency Ultrasound in the Diagnosis of Anal Fistula. Anhui Medical Journal, 21, 1793-1795.

[5] Liu, F. (2013) Clinical Application of Three-Dimensional Reconstruction of Intraluminal Ultrasound before Complex anal Fistula Surgery. Chinese Medical Guide, 11, 96-97.

[6] Zhang, Y., Wang, Y. and Wang, M.L. (2016) Three-Dimensional Volumetric Ultrasound Combined with Color Doppler Technology in the Development of Normal Fetal Aortic Arch and Head and Arm Vessels Application in Examination. Journal of China Medical University, 45, 531-534.

[7] Wu, B.S., Guo, J.Y. and Wang, J.B. (2018) Advances in the Treatment of Perianal Abscess and Anal Fistula in Infants. People's Military Medical Sciences, 61, 278-280.

[8] Destro, F., Canazza, L., Meroni, M., et al. (2018) Tethered Cord and Anorectal Malformations: A Case Series. European Journal of Pediatric Surgery, 28, 484-490. https://doi.org/10.1055/s-0037-1606844

[9] Sarkar, A., Al Shanafey, S., Mourad, M., et al. (2018) No-Fistula vs. Fistula Type Anorectal Malformation: Outcome Comparative Study. Journal of Pediatric Surgery, 53, 1734-1736. https://doi.org/10.1016/j.jpedsurg.2018.03.014

[10] Shi, C.M. and Liu. X.Y. (2018) The Value of Three Probes in the Diagnosis of Perianal Abscess and Associated anal Fistula. Imaging Research and Medical Application, 2, 145-146.

[11] Maconi, G., Greco, M. and Asthana, A. (2017) Transperineal Ultrasound for Perianal Fistulas and Abscesses-A Systematic Review and Meta-Analysis. Ultraschall in der Medizin, 38, 265-272. https://doi.org/10.1055/s-0043-103954

[12] Kołodziejczak, M., Santoro, G., Obcowska, A., et al. (2017) Three-Dimensional Endoanal Ultrasound is Accurate and Reproducible in Determining Type and Height of Anal Fistulas. Colorectal Disease, 19, 378-384. https://doi.org/10.1111/codi.13580

[13] Zhang, Q., Lu, J.G. and Liang, H.T. (2018) Progress in Diagnosis and Treatment of Perianal Abscess and Anal Fistula in Infants. China Medical Herald, 15, 34-37. 